\title{
Non-rigid Registration of 3D Multi-channel Microscopy Images of Cell Nuclei
}

\author{
Siwei Yang ${ }^{1}$, Daniela Köhler ${ }^{2}$, Kathrin Teller ${ }^{2}$, Thomas Cremer ${ }^{2}$, \\ Patricia Le Baccon ${ }^{3}$, Edith Heard ${ }^{3}$, Roland Eils ${ }^{1}$, and Karl Rohr ${ }^{1}$ \\ ${ }^{1}$ Biomedical Computer Vision Group, Dept. Theoretical Bioinformatics, DKFZ \\ Heidelberg, and University of Heidelberg, IPMB, Dept. Bioinformatics and \\ Functional Genomics, Im Neuenheimer Feld 364, D-69120 Heidelberg, Germany \\ s.yang@dkfz.de \\ ${ }^{2}$ Ludwig Maximilians University Munich, Biozentrum, Dept. of Biology II, \\ Anthropology and Human Genetics, Germany \\ ${ }^{3}$ CNRS UMR 218, Curie Institute, 26 rue d'Ulm, 75248 Paris Cedex 05, France
}

\begin{abstract}
We present an intensity-based non-rigid registration approach for normalizing 3D multi-channel microscopy images of cell nuclei. A main problem with cell nuclei images is that the intensity structure of different nuclei differs very much, thus an intensity-based registration scheme cannot be used directly. Instead, we first perform a segmentation of the images, smooth them by a Gaussian filter, and then apply an intensitybased algorithm. To improve the convergence rate of the algorithm, we propose an adaptive step length optimization scheme and also employ a multi-resolution scheme. Our approach has been successfully applied using $2 \mathrm{D}$ cell-like synthetic images, 3D phantom images as well as 3D multichannel microscopy images representing different chromosome territories and gene regions (BACs). We also describe an extension of our approach which is applied for the registration of $3 \mathrm{D}+\mathrm{t}(4 \mathrm{D})$ image series of moving cell nuclei.
\end{abstract}

\section{Introduction}

For building maps of the 3D structure of chromatin fiber of the human genome in the cellular interphase, different cell nuclei need to be evaluated. The aim is to relate geometric information to genome function to obtain a better understanding of how chromatin structure affects gene regulation in normal as well as abnormal cells. Hence, one of the essential tasks is to analyze a large amount of multi-channel 3D microscopy images, which represent cell nuclei as well as genomic structures like chromosome territories and gene regions (BACs). However, since nuclei of different cells are used it is necessary to normalize these images, i.e., we have to put them into the same coordinate system. Normalization can be achieved by image registration and generally one has to cope with both global rigid transformations as well as local non-rigid deformations.

Non-rigid registration approaches can be divided in two main categories: geometry-based and intensity-based schemes. With geometry-based approaches 
first geometric structures such as points or surfaces are extracted from the images and based on that a non-rigid transformation is computed. One main difficulty is the finding of correspondences which often requires some kind of user-interaction. Intensity-based approaches, on the other hand, directly work on the image intensities and have the advantage that they are fully automatic. Recently, also hybrid approaches based on both geometry and intensity information have been proposed. The above-mentioned types of approaches have been widely applied in medical image analysis (see [1] for recent survey), however, application to biological image data is rare. According to our knowledge non-rigid registration schemes have not yet been used for 3D multi-channel microscopy images of different cell nuclei. An approach for aligning images of the same cell in different states has been described in 2. For rigid registration of 3D cell images we refer to 3 .

We have developed an intensity-based approach for non-rigid registration of 3D multi-channel microscopy images of different cell nuclei. Our approach is based on the demons algorithm [4, which has been successfully applied in medical image analysis. However, a main problem with $3 \mathrm{D}$ cell nuclei images is that the intensity structure of different nuclei differs very much, and thus an intensitybased scheme cannot be used directly. Therefore, in contrast to previous work, we first perform a segmentation of the images by global thresholding (after noise reduction using an anisotropic diffusion filter), smooth them by a Gaussian filter, and then apply an intensity-based algorithm. Since the computation time of the approach is relatively high, we use an adaptive step length optimization algorithm as well as a multi-resolution scheme which improves the speed significantly. Our approach is fully automatic and has been successfully applied using 2D cell-like synthetic images, 3D phantom images as well as 12 different 3D multi-channel microscopy images of cell nuclei. We also present an extension of our approach, which is applied for the registration of 3D temproal image series (4D datasets) of cell nuclei.

\section{Non-rigid Registration of 3D Cell Nuclei}

\subsection{Preprocessing and Rigid Registration}

To register 3D multi-channel microscopy images of cell nuclei we first reduce the noise in the images by applying a structure-preserving anisotropic diffusion filter [5]. Then we segment the 3D images by a global thresholding scheme, which is possible since the contrast in the images is relatively large. Afterwards we apply a rigid registration scheme. We have performed a systematic study of rigid registration algorithms for $2 \mathrm{D}$ and $3 \mathrm{D}$ images. We have tested different similarity metrics (mean squared error, normalized correlation coefficient, mutual information) and two different optimizers (regular gradient descent optimizer and Powell optimizer) 6] using 2D and 3D synthetic images, real images, and segmented images. We simulated translations and different rotations, and it turned out that the normalized correlation coefficient as similarity metric using the regular gradient descent optimizer yields the best results. 


\subsection{Non-rigid Registration of Different Cell Nuclei}

For local shape alignment after global rigid registration we employ a variant of the demons algorithm [4, which is driven by symmetric forces. With $f(\mathbf{x})$ and $g(\mathbf{x})$ representing the intensity values in the source and target image at position $\mathbf{x}$, the instantaneous deformation vector field at iteration $k$ can be written as:

$$
\begin{aligned}
d \mathbf{U}_{k}(\mathbf{x}) & =\left\{\begin{array}{cc}
\frac{2\left[f(\mathbf{x})-g\left(\mathbf{u}_{k-1}(\mathbf{x})\right)\right]\left[\nabla f(\mathbf{x})+\nabla g\left(\mathbf{u}_{k-1}(\mathbf{x})\right)\right]}{p(\mathbf{x})} & p(\mathbf{x}) \geq \epsilon \\
0 & p(\mathbf{x})<\epsilon
\end{array}\right. \\
p(\mathbf{x}) & =\left[\nabla f(\mathbf{x})+\nabla g\left(\mathbf{u}_{k-1}(\mathbf{x})\right)\right]^{2}+\left[f(\mathbf{x})-g\left(\mathbf{u}_{k-1}(\mathbf{x})\right)\right]^{2}
\end{aligned}
$$

where $\nabla$ denotes the nabla operator, $\epsilon$ is a small positive constant, and $\mathbf{u}_{k}(\mathbf{x})=$ $\mathbf{x}+\mathbf{U}_{k}(\mathbf{x})$ is the transformed position $\mathbf{x}$ with the total deformation field

$$
\mathbf{U}_{k}(\mathbf{x})=\mathbf{U}_{k-1}(\mathbf{x})+d \mathbf{U}_{k}(\mathbf{x}), \quad \mathbf{U}_{0}(\mathbf{x})=\mathbf{0}
$$

Our experimental results showed that this variant using symmetric forces yields better results compared to the standard approach, where the forces are not symmetric. Application of a prior rigid transformation improves the registration result and also reduces the overall computation time. Note, that the standard demons approach assumes that the intensities remain constant (optic flow based scheme), which is not true in our application since different cell nuclei significantly differ in substructure shape and intensity. Direct application therefore results in unrealistic compression and dilation. In contrast, we here consider cell nuclei as a whole and apply the algorithm to the segmented images. However, our experiments showed that we obtain good results for the contour regions of the objects but not for the inner (homogeneous) parts. To overcome this drawback, we suggest to smooth the segmented images first using a Gaussian filter. The purpose of smoothing consists in producing larger non-zero gradient regions in the neighbourhood of edges. Typically we use a relatively large value of the standard deviation of the Gaussian leading to strong smoothing $(\sigma=6)$. The deformation field obtained in this way is more homogeneous and appears to be more correct.

Adaptive step length optimization scheme. One problem still is the relatively high computation time of the algorithm, which is typical for intensitybased non-rigid registration schemes. For 3D images typically several hours are required. To accelarate the algorithm, we propose an adaptive step length optimization scheme which is described in the following. We note, that the demons algorithm can be interpreted as an iterative optimizing approach, where the step lengths during optimization are determined based on (11). However, in practise often the computed step lengths are too small and therefore many iterations are necessary to find the solution. We suggest to increase the step lengths and automatically adapt them during optimization. For adaptation we use a scheme which is similar to binary search [7] and we apply it for each voxel of an image during optimization. Binary search is a search technique for finding a particular 
value in a list and it is an example of a divide-and-conquer algorithm. It is known that binary search is much faster than linear search.

While standard binary search is used to find a specific value in an order list of values, in our case we want to find the voxels in the target image whose intensities are most similiar to that of the considered voxels in the source image. Note that, in our application we can assume that there is only one object in an image (the cell nucleus) and for registration we use the segmented and Gaussian smoothed images. Therefore, the intensity values in the image are always increasing from the outside to the inner part of the segmented object, and thus are also sorted. Consequently, if we calculate the difference image between two images to be registered then this gives an indication of how good the chosen step lengths are. In our optimization algorithm we exploit this fact. If at a certain voxel there is a sign change of the difference image in subsequent iteration steps then the step length was chosen too large and the search exceeds the target position. At the beginning of the iteration the step lengths $\alpha_{0}(\mathbf{x})$ are determined based on the intensity difference between the two images $f(\mathbf{x})$ and $g(\mathbf{x})$ normalized by the maximal intensity value $I_{\max }$ :

$$
\alpha_{0}(\mathbf{x})=\frac{c[f(\mathbf{x})-g(\mathbf{x})]}{I_{\max }}
$$

where $c$ denotes a constant larger than 1 and determines the factor of the step lengths at the beginning of the iteration. For 8 bit images we have $I_{\max }=255$. Because of the normalization the values for the ratio in (4) (without considering c) lie between -1 and 1 . It is obvious that the larger the geometric difference between two images, i.e., the further away the contours of the objects are, the larger can the step lengths be made. However, the step lengths should not be made too large, otherwise the algorithm well exceeds the searched target position. Based on our experience $c$ should be smaller than 10, typically we used $c=4$. During the iterative optimization process we automatically adapt the step lengths $\alpha_{0}(\mathbf{x})$. Whereas with standard binary search the search interval is halved in every iteration, in our case we only halve the step length when a certain condition is fullfilled:

$$
\alpha_{k}(\mathbf{x})= \begin{cases}\alpha_{k-1}(\mathbf{x}) & \alpha_{k-1}(\mathbf{x})\left[f(\mathbf{x})-g\left(\mathbf{u}_{k-1}(\mathbf{x})\right)\right] \geq 0 \\ \frac{\alpha_{k-1}(\mathbf{x})}{2} & \alpha_{k-1}(\mathbf{x})\left[f(\mathbf{x})-g\left(\mathbf{u}_{k-1}(\mathbf{x})\right)\right]<0\end{cases}
$$

This condition has two terms. The first term is the previous (signed) step length $\alpha_{k-1}(\mathbf{x})$ and the second term is the intensity difference. If both terms have the same sign, i.e., if they are either both positive or both negative, then the search does not exceed the target position yet, and we can search in the same direction with the same step length as in the previous iteration. On the other hand, if the two terms have a different sign, then the search exceeds the target position. In this case we search in the opposite direction and halve the step length. In our approach the basic equation for image registration in (1) reads:

$$
d \tilde{\mathbf{U}}_{k}(\mathbf{x})=\left|\alpha_{k}(\mathbf{x})\right| d \mathbf{U}_{k}(\mathbf{x})
$$



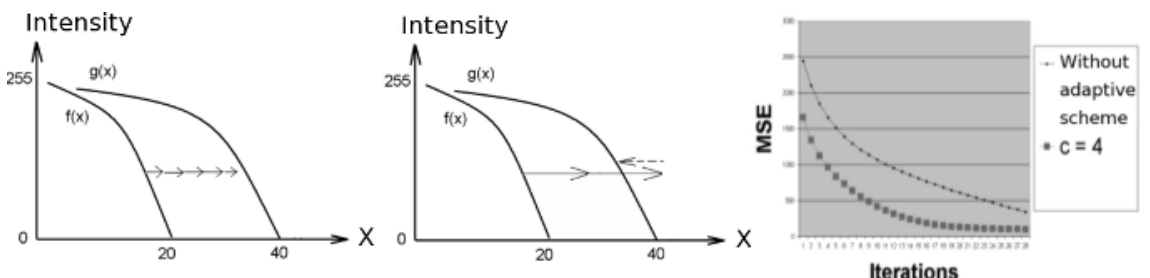

Fig. 1. Left: Optimization without adaptive step length scheme; Middle: Optimization using the adaptive step length scheme. Right: Comparison of convergence rates without and using the adaptive step length scheme $(c=4)$. MSE: mean squared error.

Note that we do not want that the algorithm is slower than the original one in case the intensity difference between two images is small. In this case $\alpha_{k}(\mathbf{x})$ is typically smaller than 1 and $d \tilde{\mathbf{U}}_{k}(\mathbf{x})$ would be smaller compared to the orignial formulation. Therefore, we impose the constraint that $\alpha_{k}(\mathbf{x})$ should always be larger or equal to 1. Thus, during iteration we use the condition $\alpha_{k}(\mathbf{x})=1$, if $\frac{\left|\alpha_{k-1}(\mathbf{x})\right|}{2}<1$. Therefore, the $\alpha_{k}$ for all voxels will finally become 1, i.e., $\lim _{k \rightarrow \infty} \alpha_{k}(\mathbf{x})=1$, and (6) converges to the original form in (1). In summary, with our algorithm large step lengths are used if there are large shape differences. On the other hand, if there are small shape differences the original equation is used. Fig. 11(left, middle) illustrates the use of the adaptive step length scheme for the $1 \mathrm{D}$ case. Without the adaptive step length scheme more than five iterations are required to align the source image $f(\mathbf{x})$ with the target image $g(\mathbf{x})$, whereas using the adaptive step length scheme needs only 3 iterations. In addition, Fig.1(right) demonstrates how the convergence rate is improved using the adaptive step length scheme $(c=4)$ in comparison to the standard approach (for the registration of $3 \mathrm{D}$ images of size $288 \times 288 \times 99$ voxels $)$.

Multiresolution approach. To further reduce the computation time we have used a multi-resolution scheme, which follows the principle "from coarse to fine". Using this scheme we could reduce the computation time, e.g., for an image of size $512 \times 512 \times 24$ voxels from $225 \mathrm{~min}$ to $74 \mathrm{~min}$ without using the adaptive step length scheme and 35 min using it. For another image of size $256 \times 256 \times 33$ voxels we could reduce the computation time from $65 \mathrm{~min}$ to $15 \mathrm{~min}$ without using the adaptive step length scheme and 9 min using it. For running the algorithm we used a Linux Server with an AMD Opteron processor of $2.4 \mathrm{GHz}$ and 7 GB memory.

For registering multi-channel 3D images we compute the transformation based on the first channel which includes the cell nuclei and then we apply the transformation to this channel as well as to the other channels which represent the different chromosome territories and gene regions (BACs).

\subsection{Non-rigid Registration of Moving Cell Nuclei}

The above described algorithm can be extended straightforwardly to be applicable to $3 \mathrm{D}+\mathrm{t}(4 \mathrm{D})$ multi-channel images of moving cell nuclei. The task here 
is the normalization of the shape of moving cell nuclei over time in order to investigate the movement of certain protein particles within the cell nuclei. Given an image series, we first perform a rigid registration between consecutive images. For example, for time instances $t$ to $t-1$ we determine the instantaneous rigid transformation matrix $d R_{t}$. The total rigid transformation matrix is then computed as the composition of previous matrices as

$$
R_{t}=d R_{1} \cdot d R_{2} \cdot \ldots \cdot d R_{t-1}
$$

Using this matrix we globally align the cell nucleus at each time point $t$ w.r.t the first time point $t_{0}$. Second, we perform a non-rigid registration by determining the deformation fields for consecutive images, e.g., $\mathbf{U}_{t}(\mathbf{x})$ from time point $t$ to $t-1$. The total deformation field is then calculated by composing these deformation fields. In addition, we regularize the composed deformation field by applying a Gaussian filter $G(\mathbf{x})$.

$$
\begin{array}{r}
\mathbf{U}_{t}(\mathbf{x})=d \mathbf{U}_{t-1}(\mathbf{x}) \circ \ldots \circ d \mathbf{U}_{2}(\mathbf{x}) \circ d \mathbf{U}_{1}(\mathbf{x}) \\
d \mathbf{U}_{t}(\mathbf{x}) \circ d \mathbf{U}_{t-1}(\mathbf{x}) \rightarrow G(\mathbf{x}) *\left[d \mathbf{U}_{t}(\mathbf{x})+d \mathbf{U}_{t-1}(\mathbf{x})\right]
\end{array}
$$

To speed up the calculations we use a Linux computer cluster and compute the transformations between two consecutive images in parallel.

\section{Experimental Results}

To systematically test our non-rigid registration approach we have generated 2D cell-like synthetic images. Application of the approach showed that symmetric forces lead to better results than asymmetric forces, because this variant takes into account the gradient information of both images instead of only that of one image. Next, we have validated our scheme using 3D CT images of the deformation of a silicon cube $[8$. In the cube 343 beads were embedded and it was compressed vertically. The images of the cube before and after deformation (size of images: $288 \times 288 \times 99$ voxels) and the real displacements of the 343 beads are available. We have compared the displacements computed by our approach with the real displacements at these points, and obtained an acceptable correlation coefficient of $r=0.96$ (for $12.5 \%$ strain).

Moreover, we have applied our approach to 3D multi-channel cell nucleus images representing different chromosome territories and gene regions (BACs). In total, we applied our approach to 12 multi-channel 3D images from two experiments. In the first experiment, we used 8 confocal 3D images from a 3D FISH (fluorescence in situ hybridisation) experiment with HeLa cells and using DAPI counterstain of the nucleus (image size $256 \times 256 \times 33$ voxels). We considered chromosome 4 and visualized the $3 \mathrm{D}$ registration result by overlaying the transformed segmented chromosome territories together with the surface of one of the nuclei as a reference. Fig. 2 a illustrates that before registration some chromosomes (indicated by the arrows) lie outside the reference cell nucleus. After registration with our approach, all chromosomes are inside the reference 


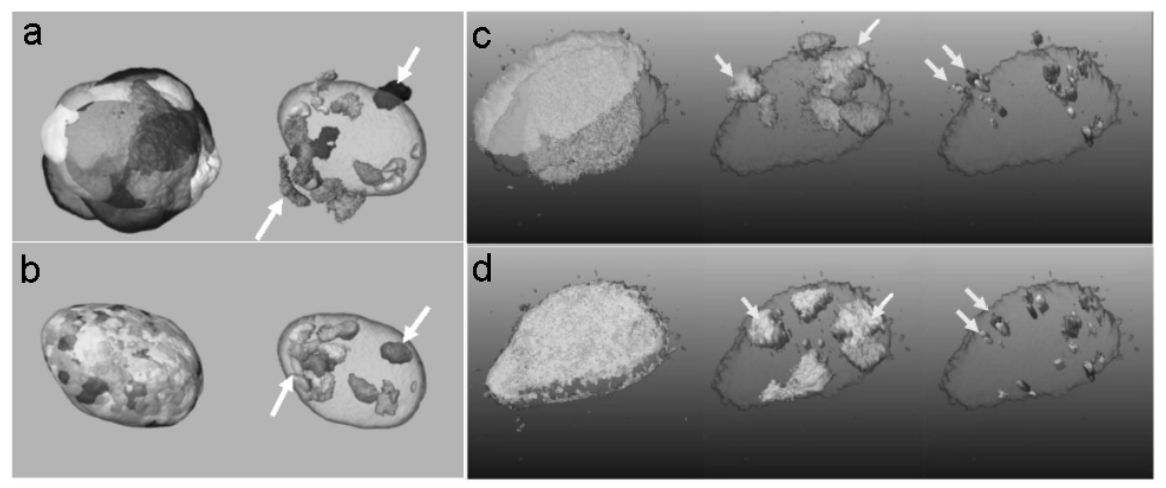

Fig. 2. a: 3D overlay of 8 different HeLa cell nuclei images (left) with chromosome 4 territories (right) before registration, b: Corresponding images after registration overlaid with a nucleus of one cell as a reference, c: 4 different HeLa cell nuclei images (left) with chromosome 2 territories (middle), and gene regions (right) before registration, d: Corresponding images after registration overlaid with a nucleus of one cell as a reference. The arrows point to corresponding chromosome territories und gene regions.

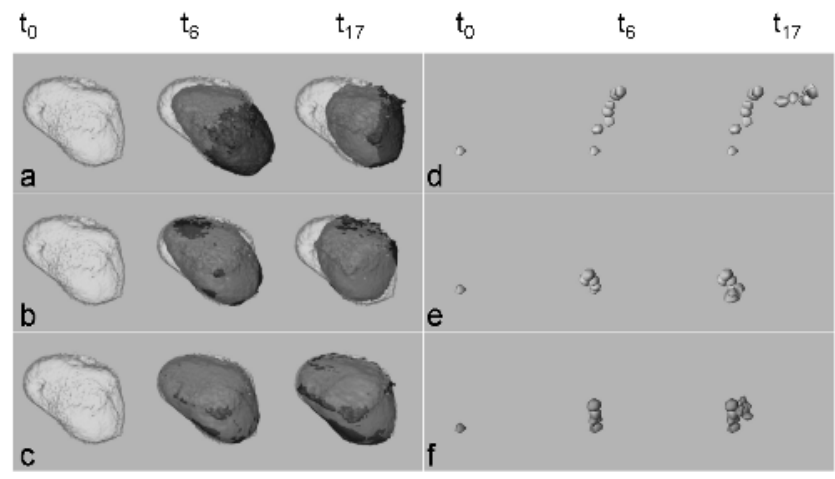

Fig. 3. $3 \mathrm{D}$ temporal image registration, selected images at time instances $t_{0}, t_{6}, t_{17}$, a: $3 \mathrm{D}$ view of one cell nucleus (white) as fixed reference nucleus $\left(t_{0}\right)$, and moving nucleus over time (black), b: After rigid registration, c: After non-rigid registration, d: Positions of moving protein particle before registration, e: After rigid registration, f: After non-rigid registration.

cell nucleus and the chromosome structures from the different cells are located more closely together (Fig. 2 b). In this way we achieve a normalization of the image data. In the second experiment with 4 confocal 3D images (image size $512 \times 512 \times 24$ voxels), where chromosome 2 territories as well as gene regions (BACs) were stained, we obtained the results in Fig. 25, d. Again we achieve a normalization of the image data.

We have also applied our approach in a different application context, namely the registration of temporal $3 \mathrm{D}+\mathrm{t}(4 \mathrm{D})$ images series. We have applied our approach to three different images series and Fig. 3 shows the result for one 3D image series consisting of 17 images of size $256 \times 256 \times 75$ voxels. In Fig. 3 a, b,c 
are shown the original images, the result after rigid registration, and the result after non-rigid registration. In Fig. 3 d,e,f are the corresponding positions of the protein particle. It can be seen that after registration there is much less movement of the particle since the global movement of the cell nucleus has been decoupled from the movement of the particle.

\section{Summary and Discussion}

We have presented an intensity-based non-rigid registration approach for the normalization of 3D multi-channel microscopy images of cell nuclei. The approach is based on a variant of the demons algorithm, uses segmented images, and employs a multi-resolution scheme. Furthermore, to improve the convergence rate we proposed an adaptive step length optimization scheme. We have successfully applied our approach using confocal multi-channel 3D images from different FISH experiments with HeLa cells. Moreover, a straightforward extension of our approach has been successfully used to register $3 \mathrm{D}+\mathrm{t}(4 \mathrm{D})$ images of moving cell nuclei.

\section{Acknowledgement}

This work has been supported by the EU project 3DGENOME. The work benefited from the use of the Insight Toolkit (ITK) 6]. We also acknowledge the support of the Human Frontier Science Program (HFSP).

\section{References}

1. Zitova, B., Flusser, J.: Image registration methods: a survey. Image and Vision Computing 21 (2003) 977-1000

2. Mattes, J., Fieres, J., Beaudouin, J., Gerlich, D., Ellenberg, J., Eils, R.: New tools for visualization and quantification in dynamic processes: Application to the nuclear envelope dynamics during mitosis. In: Proc. MICCAI. (2001) 1323-1325

3. Rieger, B., Mollenaar, C., Dirks, R.W., van Vliet, L.J.: Alignment of the cell nucleus from labeled proteins only for $4 \mathrm{~d}$ in vivo imaging. Microscopy Research and Technique 64 (2004) 142-150

4. Thirion, J.P.: Image matching as a diffusion process: an analogy with Maxwell's demons. Medical Image Analysis 2 (1998) 243-260

5. Perona, P., Malik, J.: Scale-space and edge detection using anisotropic diffusion. IEEE Trans. on Pattern Analysis and Machine Intelligence 12 (1990) 629-639

6. Ibanez, L., Schroeder, W., Ng, L., Cates, J.: The ITK Software Guide. Kitware Inc. (2005)

7. Knuth, D.: The Art of Computer Programming, Sorting and Searching. The third edn. Volume 3. Addison-Wesley (1997)

8. Kerdok, A., Cotin, S., Ottensmeyer, M.: Truth cube: Establishing physical standards for soft tissue simulation. Medical Image Analysis 7 (2003) 283-291 